\title{
The GLAST large area telescope: Design, construction, test and calibration
}

\author{
Luca Latronico* , Gloria Spandre, On behalf of the GLAST LAT Collaboration ${ }^{1}$ \\ INFN-Pisa, L.go Buonarroti 3, 56127 Pisa, Italy
}

\begin{abstract}
The Gamma-ray Large Area Space Telescope (GLAST) is an international, multi-agency satellite mission with a vast and ambitious physics program in gamma-ray astronomy, particle astrophysics and cosmology. The Large Area Telescope (LAT) is the main instrument onboard GLAST and will detect high energy gamma rays with unprecedented resolution and sensitivity. After successful integration of the three detector subsystems - a silicon-strip tracker-converter, a CsI imaging calorimeter and an outer plastic scintillator serving as anti-coincidence detector-into the telescope, the LAT was installed on the satellite in preparation for the January 2008 launch. Highlights of the LAT instrument performance and of the main technological challenges encountered during the telescope design, commissioning and calibration phases are discussed here, as well as their impact on the mission discovery potential.
\end{abstract}

\section{Introduction}

The GLAST [1] Large Area Telescope (LAT) is a unique $\gamma$-ray observatory capable of scanning the whole sky in a few hours, building spectra over four energy decades $(20 \mathrm{MeV}$ to $\geqslant 300 \mathrm{GeV})$ with $\leqslant 10 \%$ energy resolution and locating sources down to arcmin level. LAT data will cover the existing gap in the observations from previous generation $\gamma$-ray satellites, like EGRET [6], and the most modern ground imaging Cherenkov detectors, like HESS [7] and MAGIC [8]. The high sensitivity of the telescope will not only allow detection of thousands of new sources and possibly new classes of sources, but coupled to a high source localization power and energy resolution, it will greatly improve the capabilities of testing different models of the physical processes that power celestial sources like Active Galactic Nuclei, Supernova Remnants, Black Holes, Pulsars and sources of primary cosmic rays (CR).

The LAT is a pair-conversion telescope where $\gamma$-rays convert into $\mathrm{e}^{+} / \mathrm{e}^{-}$pairs in a high resolution tracker-

\footnotetext{
*Corresponding author.

E-mail address: luca.latronico@pi.infn.it (L. Latronico).

${ }^{1} \mathrm{http}: / /$ www-glast.stanford.edu
}

converter, which consists of a sequence of silicon-strip detectors and tungsten layers; electron energies are measured in a CsI imaging calorimeter placed downstream of the tracker. Rejection of charged-particle background is achieved by an external, segmented anti-coincidence shield built out of plastic scintillators.

Design, construction, testing and calibration of the LAT instrument have combined technologies, methods, institutions and dedication from both the high-energy physics and the $\gamma$-ray astronomy communities. The result is a very efficient, accurate, reliable and durable observatory for $\gamma$-ray physics, that meets all the science performance requirements, as shown in Table 1.

\section{Design and construction of the LAT detector}

The three main detectors of the LAT were developed in parallel, with common design principles imposed by satellite operation such as high modularity, readout redundancy, low mass and power consumption, absence of consumables, extreme mechanical stiffness. The LAT itself is a modular instrument composed of an array of $4 \times 4$ identical towers, each comprising a tracker, a 
Table 1

LAT performance table $(\perp$ stands for on-axis)

\begin{tabular}{|c|c|c|}
\hline Parameter & Requirement & $\begin{array}{l}\text { Current best } \\
\text { estimate }\end{array}$ \\
\hline Effective Area & $>8000 \mathrm{~cm}^{2}$ & $9000 \mathrm{~cm}^{2}$ \\
\hline $\begin{array}{l}\text { En. Resolution } \\
(100 \mathrm{MeV}, \perp)\end{array}$ & $<10 \%$ & $\sim 10 \%$ \\
\hline En. Resolution $(10 \mathrm{GeV})$ & $<10 \%$ & $<6 \%$ \\
\hline $\begin{array}{l}\text { En. Resolution } \\
(10-300 \mathrm{GeV}, \perp)\end{array}$ & $<20 \%$ & $<8 \%$ \\
\hline $\begin{array}{l}\text { Angular Resolution } \\
(100 \mathrm{MeV}, \perp)\end{array}$ & $<3.5^{\circ}$ & $<3.2^{\circ}$ \\
\hline $\begin{array}{l}\text { Angular Resolution } \\
(10 \mathrm{GeV}, \perp)\end{array}$ & $<0.15^{\circ}$ & $<0.1^{\circ}$ \\
\hline Point Source Sensitivity & $<6 \times 10^{-9} \mathrm{~cm}^{-2} \mathrm{~s}^{-1}$ & $<4 \times 10^{-9} \mathrm{~cm}^{-2} \mathrm{~s}^{-1}$ \\
\hline $\begin{array}{l}\text { Source location } \\
\text { determination }\end{array}$ & $<0.5 \operatorname{arcmin}$ & $<0.4 \operatorname{arcmin}$ \\
\hline Field Of View & $>2 \mathrm{sr}$ & $>2 \mathrm{sr}$ \\
\hline Deadtime & $<100 \mu \mathrm{s}$ & $26.5 \mu \mathrm{s}$ \\
\hline
\end{tabular}

calorimeter and a custom readout electronics module. The choice of technologies was inspired by the desire to improve sensitivity and resolution to the best achievable performance while still maintaining the necessary reliability and robustness to survive the many environmental test cycles that each module had to undergo both individually and as part of the LAT.

The tracker subsystem [2] is the central detector of the LAT. It has to convert incoming photons and track the resulting $\mathrm{e}^{+} / \mathrm{e}^{-}$pairs in order to reconstruct the direction of the incoming gamma. This is achieved by stacking 36 silicon-strip detection layers in 19 mechanical units called trays, each equipped with silicon-strip sensors on both sides and readout electronics on the side, to minimize dead space between adjacent tower modules. Tungsten tiles are placed at the bottom side of each tray just above the silicon sensors in order to place the position sensitive layers as close as possible to the converter and minimize the effect of multiple scattering. A variable thickness of the tungsten layer, with thicker tiles at the bottom, was chosen to achieve a trade-off between resolution and conversion efficiency. With its $74 \mathrm{~m}^{2}$ of silicon and almost $900 \mathrm{~K}$ channels, the GLAST tracker is the largest silicon-strip detector ever built for space applications. The tracker also provides the principal trigger signal for the LAT and is capable of working at $10 \mathrm{kHz}$ with minimal dead time. The average hit efficiency is $>99.5 \%$ at $\frac{1}{4}$ MIP nominal threshold, with a single strip noise occupancy $<10^{-6}$ and a dead-channel fraction $<0.2 \%$. The total power consumption of the whole tracker system is below $160 \mathrm{~W}$. All 17 flight units and a beam test unit were assembled and tested in less than one year. A reliable industrial production of Si-strip sensors, a fully custom, digital readout electronics and light, stiff carbon composite mechanical structures are the main technologies allowing such a performance.
The calorimeter subsystem [3] modules are stacks of $\mathrm{CsI}(\mathrm{Tl})$ crystals arranged in a hodoscopic configuration of eight layers of 12 columns each, for a total of 8.3 radiation lengths. Each Crystal Detector Element (CDE) is assembled by bonding PIN diodes on each side of a CsI log, so that the CDE scintillation light is read out at both ends. The sum of the signals provides the energy deposited in the crystal while the ratio is a measure of the location of the energy deposition along the crystal. Each photodiode is processed by an electronics chain with preamplifier, shaper and dual track-and-hold. The four-channel readout of each crystal end can then support the large $2 \mathrm{MeV}-60 \mathrm{GeV}$ dynamic range imposed by the science performance requirements making use of the different available gain ratios spanning 512:1. An analog multiplexer supplies these four signals to a single 12-bit ADC, and programmable range selection logic selects the lowest unsaturated energy range for readout. Each diode is also equipped with a parallel, fast shaper that provides a trigger signal whenever a crystal records energy above some threshold. The logical OR of the low and high energy discriminators from the CDEs in each layer form the CAL-LO and CAL-HI trigger primitives, respectively, with nominal flight thresholds of $100 \mathrm{MeV}$ and $1 \mathrm{GeV}$ per crystal. Twenty CAL modules were assembled in 2004-2005, including 16 flight units, two flight spares, an Engineering Model, and a beamtest unit.

The anti-coincidence subsystem [4] is the outermost LAT detector and is responsible for vetoing the background of charged CR (mainly protons and electrons), which is 3-5 orders of magnitude more intense than the $\gamma$-rays the LAT should identify. To provide a high detection efficiency $\left(0.9997\right.$ over a total active area of $\left.8.3 \mathrm{~m}^{2}\right)$ and reduce signal fluctuations, $1 \mathrm{~cm}$ thick scintillator tiles were chosen, with wavelength-shifting fibers spaced at $5 \mathrm{~mm}$ to bring the signal to the periphery readout electronics. To suppress the self-veto caused by backsplash from high-energy particles hitting the calorimeter, the ACD is segmented into 89 tiles and ACD hits far from the reconstructed point of entry are ignored. The hermeticity of the ACD is enhanced by tiles overlapping in one dimension and use of scintillating fiber ribbons in the gaps. The ACD integration and environmental test were completed in 2005.

\section{Integration and test of the LAT}

Integration of the flight LAT modules took place at SLAC in parallel to the construction, test and qualification of the subsystems. It was completed in December 2005 with the ACD integration, about one year after the arrival of the first flight units. The integrated LAT was then moved to Naval Research Laboratory for a complete observatory level environmental test cycle (EMI-EMC, vibrational and thermal-vacuum tests), which was successfully completed in September 2006. Performance verification was ensured by charge injection and CR measurements at all stages during integration and environmental tests. Only three 


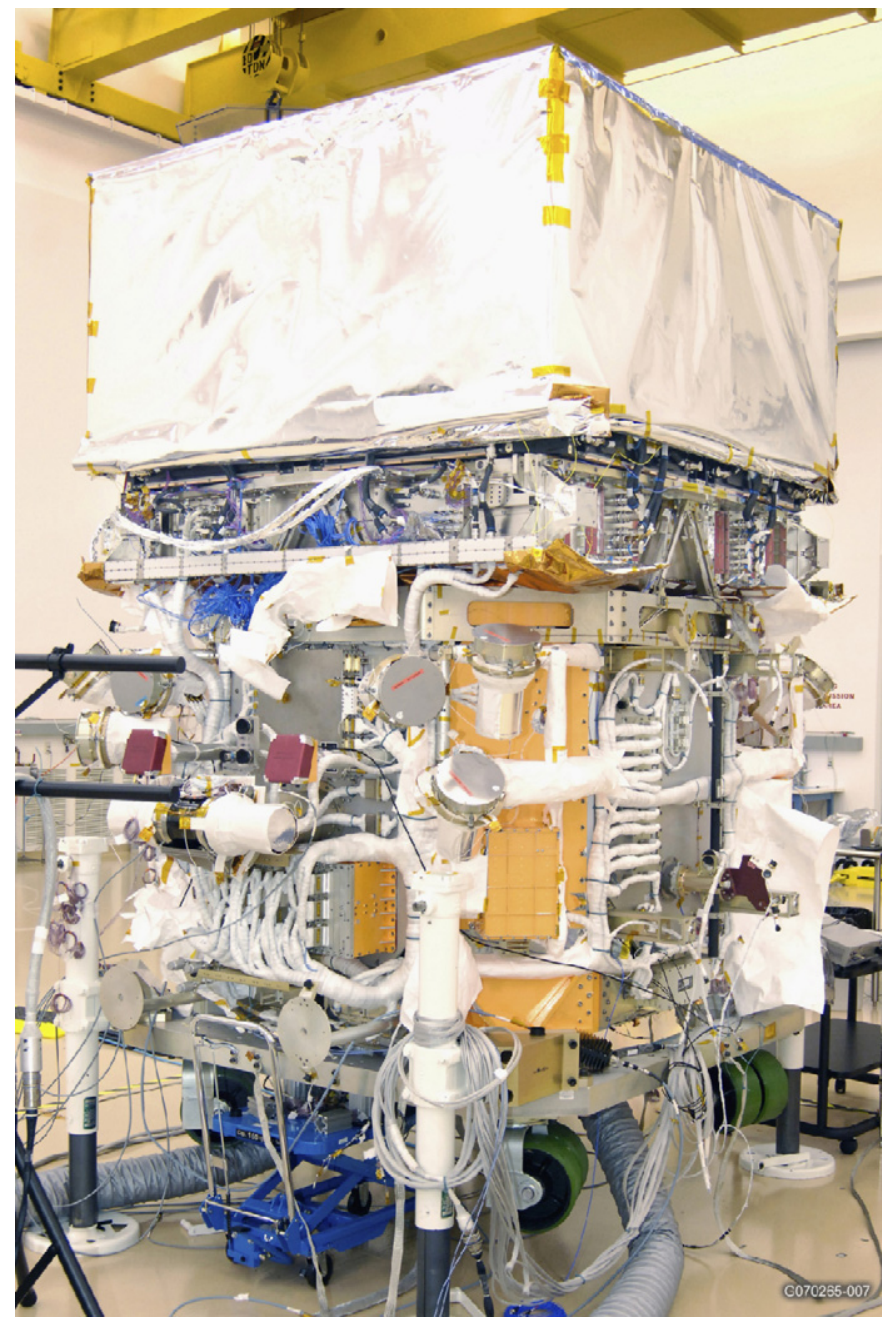

Fig. 1. The GLAST observatory at General Dynamics, December 2006.

months afterwards the LAT was integrated onto the satellite at General Dynamics, together with the Gamma Burst Monitor (GBM) [9], the other detector onboard the GLAST satellite, which is designed for fast detection and localization of gamma-ray bursts in the $10 \mathrm{keV}-25 \mathrm{MeV}$ energy band. The GLAST observatory (see Fig. 1) will go through environmental tests in the summer of 2007 and be ready for launch in December of the same year.

\section{Calibration of the LAT}

The LAT calibration on the ground combines analysis of cosmic ray data with measurements in particle beams from accelerators. An advanced Monte Carlo simulation of the LAT, based on the Geant4 package, was set up to reproduce the LAT response to such radiation and to benchmark the event reconstruction and the background rejection strategy before launch and during operation. To validate the LAT simulation, a massive campaign of beam tests was performed between July and November 2006, in parallel with the LAT integration and test, on the LAT

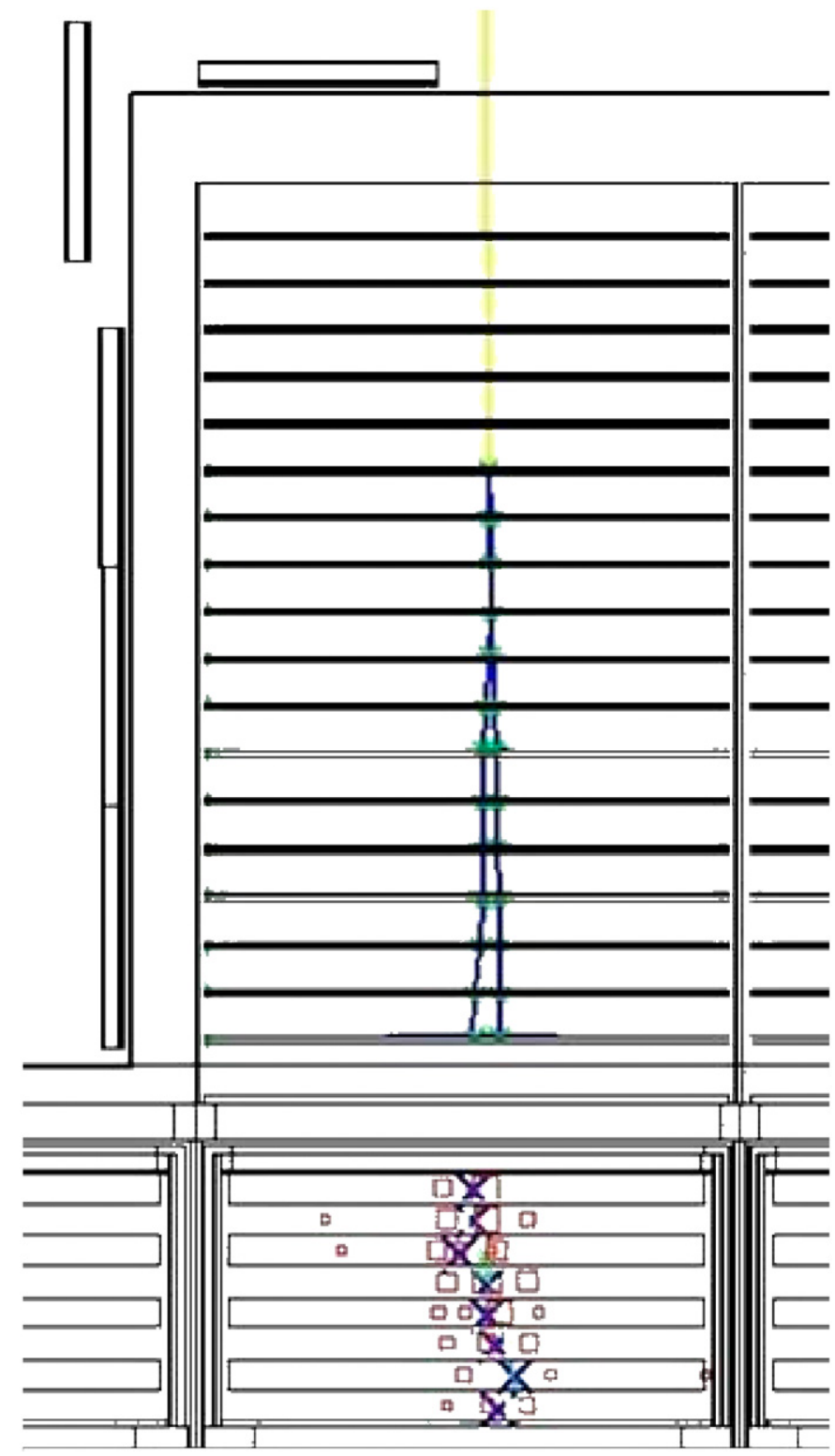

Fig. 2. A $470 \mathrm{MeV} \gamma$-ray from the CERN beam test enters the CU and converts in the sixth tungsten foil; the $\mathrm{e}^{-}$and $\mathrm{e}^{+}$are tracked and their energy is measured in the calorimeter.

Calibration Unit (CU). This is a detector built with spare flight modules, which was exposed to a large variety of beams from the CERN and the GSI accelerator facilities, representing the whole spectrum of the signal that the LAT will detect. Beams of photons $(0-2.5 \mathrm{GeV}$; see Fig. 2), electrons $(1-300 \mathrm{GeV})$, hadrons $(\pi$ and $p, 5 \mathrm{GeV}-100 \mathrm{GeV})$ and ions $(\mathrm{C}, \mathrm{Xe}, 1.5 \mathrm{GeV} / n)$ were shot through the $\mathrm{CU}$ to measure the physical processes taking place in the detector and eventually fine-tune their description in the LAT Monte Carlo simulation. Preliminary results indicate that the current Monte Carlo simulation reproduces well the angular resolution of the Tracker, the electro-magnetic shower shape in the Calorimeter and the backsplash signal in the Anti-Coincidence Detector [5]. 


\section{References}

[1] N. Gehrels, P.F. Michelson, Astropart. Phys. 11 (1999) 277.

[2] H. Tajima, Nucl. Instr. and Meth. A 569 (2006) 140.

[3] A. Chekhtman, AIP Conf. Proc. Ser. 867 (2006) 151.

[4] A.A. Moiseev et al., Astropart. Phys. 27 (2007) 339.
[5] L. Baldini et al., Proceedings of the First GLAST Symposium, 5-8 February 2007, Stanford University, AIP Conf. Proc. Series, in press.

[6] D.J. Thompson, et al., ApJL 86 (1993) 629.

[7] J. Hinton, New Astron. Rev. 48 (2004) 331.

[8] J.A. Barrio et al., MPI-PhE/98-5, 1998.

[9] A. Von Kielnin, ESA SP-459 (2001) 529. 